\title{
SUPERVIVENCIA Y CRECIMIENTO INICIAL DE PLANTAS MICROPROPAGADAS DE RAULÍ (Nothofagus alpina (Poepp et Endl) Oerst) ESTABLECIDAS EN ENSAYOS CLONALES
}

\author{
Braulio Gutiérrez C. ${ }^{1}$
}

\section{RESUMEN}

Se evaluó el desempeño en terreno a los tres años de edad de plantas micropropagadas de raulí, en tres ensayos clonales establecidos en Chile, en sitios representativos de la distribución natural de la especie, en las provincias de Malleco, Cautín y Valdivia, entre los 800 y $1.000 \mathrm{msnm}$.

Se observó un comportamiento superior de los clones micropropagados en relación a los testigos provenientes de semilla comercial, situación esperable debido al origen selecto de los clones, los cuales corresponden a réplicas vegetativas de árboles plus rigurosamente seleccionados en función de sus características de volumen y forma. Aún así, se observa también una gran variación entre clones y entre las procedencias de los mismos, así como evidencias de interacción genotipo ambiente.

Palabras clave: Nothofagus alpina, silvicultura clonal.

\section{SUMMARY}

The field performance at three years old of micropropagated plants of Raulí, established in three clonal tests in representative sites of the natural distribution of the species in Chile (Malleco, Cautín and Valdivia provinces, between 800 and 1,000 masl) was analyzed.

A superior behavior of the micropropagated clones in relation to the control plants originated from commercial seed was observed, expectable situation due to selected origin of the clones, which are vegetative copies of rigorously selected plus trees based on their characteristics of volume and forms. A great variation between clones and between theirs provenances was also observed, as well as evidences of genotype environment interaction.

Keywords: Nothofagus alpina, clonal forestry.

1-Ingeniero Forestal. Instituto Forestal Sede Bio Bio. Chile. E-mail: bgutierr@infor.cl 


\section{INTRODUCCIÓN}

Raulí (Nothofagus alpina (Poepp et Endl.) Oerst) es una especie forestal nativa de los bosques de Chile y Argentina, que en el país ha sido definida como una especie prioritaria para contribuir a la diversificación de las plantaciones forestales nacionales y que como recurso ha sido fuertemente degradado debido a la cosecha indiscriminada de que ha sido objeto como consecuencia de la alta calidad y valor de su madera.

El tradicional e histórico "floreo" (extracción selectiva) sufrido por los bosques en que se presenta raulí ha reducido considerablemente la proporción de individuos de alta calidad maderera. Consecuentemente, INFOR inició en 1996 un programa de mejoramiento genético orientado a recuperar y mejorar el potencial productivo de esta especie. Entre otras actividades, durante este programa inicial se identificaron árboles plus, los que fueron rigurosamente seleccionados en función de su superioridad en volumen y forma. Posteriormente, a partir del año 2001, un nuevo proyecto de investigación permitió desarrollar procedimientos para la multiplicación clonal de los árboles selectos, mediante técnicas de cultivo in vitro. El material multiplicado en esta iniciativa, rigurosamente identificado por clon y lugar de procedencia, fue establecido en ensayos clonales en terreno con la finalidad de evaluar el desempeño de los clones y diferenciar el material genético idóneo para distintas zonas de plantación dentro del área de distribución natural de raulí

La descripción de los ensayos mencionados y los resultados de su evaluación de crecimiento y supervivencia a los tres años de edad se describen en el presente documento. La información entregada en este análisis será de gran valor para orientar decisiones respecto a la conveniencia de usar clones selectos, qué material es más adecuado para cada área geográfica y qué comportamiento inicial podrá esperarse de sus plantaciones operacionales. Los clones considerados constituyen un material genético de alto valor, especialmente apropiado para establecer plantaciones o contribuir al enriquecimiento de bosques degradados, aspecto que cobra especial relevancia en la actualidad, en el marco de la nueva ley de recuperación del bosque nativo.

\section{OBJETIVOS}

Evaluar el desempeño inicial de tres ensayos clonales de raulí para identificar los clones de mejor desempeño en distintas áreas geográficas representativas de la distribución natural de la especie.

\section{MATERIAL Y MÉTODO}

Se evalúan tres ensayos clonales de raulí identificados con el nombre del predio donde fueron establecidos, Reserva Forestal Malleco, Molulco y Remeco (Cuadro N 1). 


\section{Cuadro $\mathrm{N}^{\circ} 1$ \\ ENSAYOS CONSIDERADOS EN LA EVALUACIÓN}

\begin{tabular}{|l|l|l|l|l|l|}
\hline Ensayo & $\begin{array}{l}\text { Provincia } \\
\text { Región }\end{array}$ & Comuna & $\begin{array}{l}\text { Altitud } \\
(\mathbf{m s n m})\end{array}$ & Propietario & Establecimiento \\
\hline RF Malleco & $\begin{array}{l}\text { Malleco } \\
\text { La Araucanía }\end{array}$ & Collipulli & 980 & CONAF & Oct. 2005 \\
\hline Molulco & $\begin{array}{l}\text { Cautín } \\
\text { La Araucanía }\end{array}$ & Melipeuco & 800 & MAGASA & Ago. 2005 \\
\hline Remeco & $\begin{array}{l}\text { Valdivia } \\
\text { Los Ríos }\end{array}$ & Panguipulli & 870 & $\begin{array}{l}\text { Forestal } \\
\text { Neltume } \\
\text { Carranco }\end{array}$ & Oct. 2005 \\
\hline
\end{tabular}

En la selección de terrenos para los ensayos clonales de raulí, se utilizó un sistema de clasificación de sitios desarrollado para Chile por Schlatter et al. (1994; 1995). Como base se usó la distribución natural de Nothofagus alpina. Se usó el material cartográfico base de la distribución natural de los tipos forestales Roble-Raulí-Coigüe y Coigüe-RaulíTepa, a escala 1:250.000, para las provincias de Malleco, Cautín y Valdivia. Además, se utilizó la información del sistema de clasificación de Schlatter y Gerding (1995), también a escala 1:250.000. Luego, para poder definir las zonas y distritos de crecimiento a los cuales están suscritas las áreas elegidas como representativas de Nothofagus alpina, se traslaparon ambas coberturas. El trabajo de selección y caracterización de los sitios fue efectuado por el mismo autor principal de los trabajos mencionados. Las áreas con raulí de la Cordillera de la Costa no fueron consideradas en la selección de sitios, debido a que las poblaciones principales se encuentran en la Cordillera de los Andes y a que los sitios de la Cordillera de la Costa son de menor interés productivo para la especie.

La descripción de los sitios de cada ensayo es la siguiente (Schlatter y Steuer, 2005):

Ensayo RF Malleco: Ladera alta a media, en loma con exposición Sur, a 980 msnm., con 40 a $50 \%$ de pendiente, convexa pero con contorno recto (levemente convexo). El suelo pertenece la serie Santa Bárbara, es de cenizas volcánicas modernas, con presencia de pumicita. El drenaje es rápido y las raíces se extienden por sobre los 100 $\mathrm{cm}$. El sitio presenta un estrato arbóreo dominante de raulí, originado por rebrote, y coigüe, y codominante de tepa y avellanillo. El bosque es bastante abierto y fue afectado por sucesivos incendios, además de una intensa explotación maderera. Presenta una precipitación entre 2.500 y $3.000 \mathrm{~mm}$ anuales.

Ensayo Molulco: Ladera media, de exposición Sur, colindante a un escarpe rocoso, dentro de una colina, con elevación de $800 \mathrm{msnm}$. La ladera es recta a cóncava y su contorno cóncavo a recto. El suelo pertenece a la serie Huerere, es de cenizas volcánicas, las que cubren un suelo enterrado, presenta una capa de pumicita delgada dentro del perfil. Las raíces se extienden por sobre los $100 \mathrm{~cm}$, pero la principal masa se presenta hasta los $90 \mathrm{~cm}$, el drenaje interno es rápido. El sitio está cubierto de un renoval abierto, con un estrato dominante de roble y raulí, y como acompañantes 
tepa y lleuque. El área ha sido intervenida para pastoreo de ganado. Presenta una precipitación aproximada de $3.000 \mathrm{~mm}$ anuales.

Ensayo Remeco: Ladera baja en sector de colinas, con exposición Oeste y elevación de $870 \mathrm{msnm}$. La pendiente es de 35 \%, recta a cóncava con contorno levemente convexo. El suelo está en la transición entre las series Liquiñe y Ralún, es de cenizas volcánicas estratificadas, con presencia de pumicita. Las raíces se extienden por sobre los $150 \mathrm{~cm}$, su drenaje interno es rápido a moderado. Se encuentra alejado del efecto inmediato del volcán Choshuenco, lo que explica el predominio de cenizas y arenas pumicíticas y la ausencia de escorias volcánicas El estrato arbóreo remanente lo conforman coigüe y raulí, como dominantes, y tepa y mañío, como codominantes, en un bosque muy floreado e invadido por coligüe. La precipitación media anual es superior a los 4.000 $\mathrm{mm}$.

Los tres ensayos poseen el mismo diseño experimental. Corresponden a plantaciones establecidas con réplicas micropropagadas de árboles plus (clones), distribuidas en cuatro bloques al azar, dentro de los cuales cada clon se representa por una parcela lineal de seis plantas (rametos). Cada ensayo comprende del orden de 20 clones representado por 6 rametos en cada uno de sus cuatro bloques. Como testigos se consideran 4 lotes de plantas de raulí originadas de semillas. La descripción del material genético considerado en cada ensayo se detalla en el Cuadro $N^{\circ} 2$ (Gutiérrez et al., 2005).

Cada clon considerado en los ensayos corresponde a un árbol único, identificado mediante un código que le fue asignado al momento de su selección y que se ha mantenido hasta la fecha, permitiendo conocer con precisión el origen de cada una de las plantas producidas y representadas en los ensayos clonales.

La medición, correspondiente al tercer año de establecidos los ensayos, se digitó para construir una base de datos en que se registró la identificación y procedencia de cada clon junto con información de su crecimiento en altura, supervivencia y estado de las plantas. La base de datos de cada ensayo se evaluó en forma individual y también las tres en forma conjunta. Se efectuó análisis de varianza y prueba de comparaciones múltiples de medias (Scott y Knott, $\alpha=0,05)$ para las variables altura y supervivencia, utilizando el programa estadístico InfoStat, agrupando la información tanto por clon como por origen (procedencia). 


\section{Cuadro $\mathrm{N}^{\circ} 2$}

MATERIAL GENÉTICO CONSIDERADO EN LOS TRES ENSAYOS CLONALES DE RAULÍ

\begin{tabular}{|c|c|c|c|c|c|}
\hline \multirow{2}{*}{$\begin{array}{l}\text { Código } \\
\text { Clon }\end{array}$} & \multirow{2}{*}{ Origen } & \multirow{2}{*}{$\begin{array}{l}\text { Identificación } \\
\text { De terreno }\end{array}$} & \multicolumn{3}{|c|}{ Ensayos } \\
\hline & & & RF Malleco & Molulco & Remeco \\
\hline 3 & Melipeuco & Melipeuco 3 & $x$ & & $x$ \\
\hline 7 & Melipeuco & Melipeuco 8 & $x$ & $x$ & $x$ \\
\hline 12 & Jauja & Jauja 7 & $x$ & $x$ & $x$ \\
\hline 23 & Voipir & Voipir 2 & $x$ & $x$ & $x$ \\
\hline 33 & Neltume & Neltume 14 & $x$ & $x$ & $x$ \\
\hline 37 & Victoria & San Gregorio 1 & $x$ & $x$ & $x$ \\
\hline 38 & Victoria & San Gregorio 2 & $x$ & $x$ & $x$ \\
\hline 40 & Victoria & San Gregorio 5 & $x$ & $x$ & $x$ \\
\hline 43 & Victoria & San Gregorio 11 & $x$ & $x$ & $x$ \\
\hline 44 & Lastarria & Lastarria 1 & $x$ & $x$ & $x$ \\
\hline 53 & Melipeuco & Melipeuco 15 & $x$ & $x$ & $x$ \\
\hline 58 & Melipeuco & Melipeuco 20 & $x$ & $x$ & $x$ \\
\hline 66 & Arquilhue & Arquilhue 4 & $x$ & $x$ & $x$ \\
\hline 88 & Curacautín & Sta. Emilia 34 & $x$ & $x$ & $x$ \\
\hline 98 & Curacautín & Manzanar 46 & & $x$ & \\
\hline 120 & HSC Huillilemu & Huillilemu clon 23 & $x$ & $x$ & $x$ \\
\hline 121 & HSC Huillilemu & Huillilemu clon 24 & $x$ & $x$ & $\mathrm{x}$ \\
\hline 131 & HSC Huillilemu & Huillilemu clon 37 & $\mathrm{x}$ & $\mathrm{x}$ & $\mathrm{x}$ \\
\hline 132 & HSC Huillilemu & Huillilemu clon 38 & $x$ & $x$ & $x$ \\
\hline 138 & HSC Huillilemu & Huillilemu clon 53 & $x$ & $x$ & $x$ \\
\hline 141 & HSC Huillilemu & Huillilemu clon 40 & $\mathrm{x}$ & $x$ & $x$ \\
\hline T1 & \multicolumn{2}{|c|}{ Plantas de semilla CEFOR } & $x$ & $x$ & $x$ \\
\hline T2 & \multicolumn{2}{|c|}{ Plantas de semilla INFOR } & $x$ & $x$ & \\
\hline T3 & \multicolumn{2}{|c|}{ Plantas de semilla INFOR con Morchella sp. } & & $x$ & \\
\hline T4 & \multicolumn{2}{|c|}{ Plantas de semilla de vivero comercial } & $x$ & $x$ & $x$ \\
\hline
\end{tabular}

Para efecto de determinar las medias de supervivencia se consideró a todas las plantas vivas, independiente de su estado, mientras que para determinar las medias de altura las bases de datos fueron depuradas usando la información de estado, para eliminar del promedio a las plantas dañadas (ápice seco, quebradas, ramoneadas, rebrotes, etc.).

\section{RESULTADOS Y DISCUSIÓN}

El Cuadro $\mathrm{N}^{\circ} 3$ resume los resultados de altura y supervivencia por ensayo, desprendiéndose como primera observación el estadísticamente significativo menor crecimiento en altura experimentado por el ensayo de la Reserva Forestal Malleco, el que a su vez obtiene el mayor valor de supervivencia y de plantas dañadas. 


\section{Cuadro $\mathrm{N}^{\circ} 3$}

RESULTADOS GENERALES DE ALTURA Y SUPERVIVENCIA POR ENSAYO

\begin{tabular}{|l|c|c|c|}
\hline Ensayo & Altura (cm) & Supervivencia (\%) & Plantas dañadas (\%) \\
\hline Remeco & 102,36 & 70,5 & 7,0 \\
\hline Molulco & 101,41 & 75,7 & 4,9 \\
\hline RF Malleco & 59,51 & 83,7 & 10.3 \\
\hline PROMEDIO & $\mathbf{8 6 , 7 7}$ & $\mathbf{7 6 , 6}$ & $\mathbf{7 . 4}$ \\
\hline
\end{tabular}

Al evaluar los ensayos en forma individual se observa que en todos ellos existen diferencias estadísticamente significativas entre los valores de altura alcanzados por cada clon (Cuadro $\mathrm{N}^{\circ} 4$ ) y origen de los mismos (Cuadro $\mathrm{N}^{\circ} 5$ ).

\section{Cuadro $\mathrm{N}^{\circ} 4$}

\section{DESEMPEÑO A NIVEL DE CLONES EN CADA UNO DE LOS ENSAYOS EVALUADOS}

\begin{tabular}{|c|c|c|c|c|c|c|c|c|c|c|c|}
\hline \multicolumn{4}{|c|}{ RF Malleco } & \multicolumn{4}{|c|}{ Molulco } & \multicolumn{4}{|c|}{ Remeco } \\
\hline Clon & $\begin{array}{l}\text { Altura } \\
(\mathrm{cm})\end{array}$ & $\left({ }^{*}\right)$ & $\begin{array}{l}\text { Sup } \\
(\%)\end{array}$ & Clon & $\begin{array}{l}\text { Altura } \\
\text { (cm) }\end{array}$ & $\left({ }^{*}\right)$ & $\begin{array}{l}\text { Sup } \\
(\%)\end{array}$ & Clon & $\begin{array}{l}\text { Altura } \\
\text { (cm) }\end{array}$ & $\left({ }^{*}\right)$ & $\begin{array}{l}\text { Sup } \\
(\%)\end{array}$ \\
\hline 7 & 91,76 & $A$ & 91,7 & T4 & 137,50 & $A$ & 8,3 & 7 & 118,53 & $A$ & 83,3 \\
\hline 12 & 75,72 & $B$ & 79,2 & 98 & 136,79 & $A$ & 100,0 & 40 & 118,40 & A & 91,7 \\
\hline 44 & 72,11 & $\mathrm{~B}$ & 100,0 & 37 & 134,35 & $\mathrm{~A}$ & 79,2 & 58 & 117,68 & $\mathrm{~A}$ & 70,8 \\
\hline 38 & 70,56 & $B$ & 83,3 & 38 & 120,09 & $\mathrm{~B}$ & 83,3 & 132 & 116,77 & $\mathrm{~A}$ & 95,8 \\
\hline T4 & 68,79 & $B$ & 54,2 & 23 & 119,53 & $B$ & 87,5 & 23 & 114,36 & $A$ & 66,7 \\
\hline 37 & 67,06 & $B$ & 100,0 & 58 & 114,90 & $\mathrm{~B}$ & 87,5 & 12 & 112,58 & A & 29,2 \\
\hline 131 & 64,22 & $\mathrm{~B}$ & 95,8 & 43 & 114,46 & $\mathrm{~B}$ & 87,5 & 33 & 109,37 & $\mathrm{~A}$ & 62,5 \\
\hline 3 & 64,14 & $B$ & 75,0 & 66 & 113,70 & $B$ & 79,2 & 53 & 105,75 & $A$ & 50,0 \\
\hline 121 & 60,57 & C & 95,8 & 138 & 111,32 & $B$ & 95,8 & 3 & 104,02 & $A$ & 50,0 \\
\hline 58 & 58,89 & $\mathrm{C}$ & 79,2 & 132 & 111,02 & $\mathrm{~B}$ & 79,2 & 43 & 103,44 & $\mathrm{~A}$ & 79,2 \\
\hline 53 & 58,11 & C & 61,1 & 121 & 110,81 & $B$ & 83,3 & 141 & 101,68 & $A$ & 83,3 \\
\hline 23 & 57,66 & $\mathrm{C}$ & 95,8 & 7 & 109,89 & $\mathrm{~B}$ & 91,7 & 38 & 100,49 & $\mathrm{~A}$ & 62,5 \\
\hline T1 & 56,77 & $\mathrm{C}$ & 45,8 & 33 & 107,49 & $B$ & 83,3 & 131 & 99,93 & $A$ & 83,3 \\
\hline 88 & 55,16 & $\mathrm{C}$ & 91,7 & 131 & 104,01 & $B$ & 75,0 & 37 & 99,77 & $\mathrm{~A}$ & 83,3 \\
\hline 141 & 52,98 & $\mathrm{C}$ & 91,7 & 12 & 96,46 & $\mathrm{C}$ & 45,8 & 138 & 99,77 & $A$ & 95,8 \\
\hline T2 & 52,15 & $\mathrm{C}$ & 70,8 & 88 & 94,66 & $\mathrm{C}$ & 66,7 & 88 & 99,59 & $A$ & 20,8 \\
\hline 40 & 51,98 & $\mathrm{C}$ & 100,0 & 141 & 92,20 & $\mathrm{C}$ & 83,3 & 44 & 94,88 & $B$ & 58,3 \\
\hline 120 & 51,13 & $\mathrm{C}$ & 100,0 & T1 & 91,67 & $\mathrm{C}$ & 79,2 & 120 & 94,42 & $B$ & 91,7 \\
\hline 132 & 51,13 & $\mathrm{C}$ & 95,8 & 53 & 88,14 & $\mathrm{C}$ & 66,7 & 66 & 91,77 & $\mathrm{~B}$ & 95,8 \\
\hline 43 & 49,98 & $\mathrm{C}$ & 91,7 & 40 & 85,55 & $\mathrm{C}$ & 79,2 & 121 & 91,64 & $\mathrm{~B}$ & 83,3 \\
\hline 66 & 49,05 & C & 79,2 & 120 & 75,89 & $\mathrm{D}$ & 79,2 & T4 & 80,67 & $\mathrm{~B}$ & 29,2 \\
\hline 138 & 43,70 & $\mathrm{C}$ & 91,7 & 44 & 72,10 & $D$ & 58,3 & T1 & 77,96 & $\mathrm{~B}$ & 75,0 \\
\hline \multirow[t]{2}{*}{33} & 41,63 & C & 83,3 & T3 & 57,97 & $\mathrm{D}$ & 79,2 & & & & \\
\hline & & & & T2 & 52,78 & $D$ & 70,8 & & & & \\
\hline Promedio & 59,51 & & 83,7 & & 101,41 & & 75,7 & & 102,36 & & 70,5 \\
\hline
\end{tabular}

$\left(^{*}\right)$ : Letras distintas en la misma columna indican diferencias estadísticamente significativas $(\alpha=0,05)$ 


\section{Cuadro $\mathrm{N}^{\circ} 5$ \\ DESEMPEÑO A NIVEL DE PROCEDENCIAS EN CADA UNO DE LOS ENSAYOS EVALUADOS}

\begin{tabular}{|l|c|c|c|c|c|c|c|c|c|}
\hline \multirow{2}{*}{ Procedencias } & \multicolumn{3}{|c|}{ RF Malleco } & \multicolumn{3}{c|}{ Molulco } & \multicolumn{3}{c|}{ Remeco } \\
\cline { 2 - 11 } & $\begin{array}{c}\text { Altura } \\
(\mathbf{c m})\end{array}$ & $\left(^{*}\right)$ & $\begin{array}{c}\text { Sup } \\
(\%)\end{array}$ & $\begin{array}{c}\text { Altura } \\
(\mathbf{c m})\end{array}$ & $\left(^{*}\right)$ & $\begin{array}{c}\text { Sup } \\
(\%)\end{array}$ & $\begin{array}{c}\text { Altura } \\
(\mathbf{c m})\end{array}$ & $\left(^{*}\right)$ & $\begin{array}{c}\text { Sup } \\
(\%)\end{array}$ \\
\hline Arquilhue & 49,05 & $\mathrm{~B}$ & 79,2 & 113,7 & $\mathrm{~A}$ & 79,2 & 91,77 & $\mathrm{~B}$ & 95,8 \\
\hline Curacautín & 55,16 & $\mathrm{~B}$ & 91,7 & 112,72 & $\mathrm{~A}$ & 81,0 & 99,59 & $\mathrm{~A}$ & 20,8 \\
\hline HSC Huillilemu & 53,96 & $\mathrm{~B}$ & 95,1 & 100,87 & $\mathrm{~B}$ & 82,6 & 100,7 & $\mathrm{~A}$ & 88,9 \\
\hline Jauja & 75,72 & $\mathrm{~A}$ & 79,2 & 96,46 & $\mathrm{~B}$ & 45,8 & 112,58 & $\mathrm{~A}$ & 29,2 \\
\hline Lastarria & 72,11 & $\mathrm{~A}$ & 100,0 & 72,1 & $\mathrm{C}$ & 58,3 & 94,88 & $\mathrm{~A}$ & 58,3 \\
\hline Melipeuco & 68,9 & $\mathrm{~A}$ & 77,8 & 104,31 & $\mathrm{~B}$ & 81,9 & 111,5 & $\mathrm{~A}$ & 63,5 \\
\hline Neltume & 41,63 & $\mathrm{~B}$ & 83,3 & 107,49 & $\mathrm{~B}$ & 83,3 & 109,37 & $\mathrm{~A}$ & 62,5 \\
\hline Victoria & 59,9 & $\mathrm{~B}$ & 93,8 & 113,61 & $\mathrm{~A}$ & 82,3 & 105,53 & $\mathrm{~A}$ & 79,2 \\
\hline Voipir & 57,66 & $\mathrm{~B}$ & 95,8 & 119,53 & $\mathrm{~A}$ & 87,5 & 114,36 & $\mathrm{~A}$ & 66,7 \\
\hline Testigos & 59,88 & $\mathrm{~B}$ & 54,2 & 72,86 & $\mathrm{C}$ & 59,4 & 79,12 & $\mathrm{~B}$ & 63,5 \\
\hline Promedio & $\mathbf{5 9 , 5 1}$ & & $\mathbf{8 3 , 7}$ & $\mathbf{1 0 1 , 4 1}$ & & $\mathbf{7 5 , 7}$ & $\mathbf{1 0 2 , 3 6}$ & & $\mathbf{7 0 , 5}$ \\
\hline
\end{tabular}

$\left.{ }^{*}\right)$ : Letras distintas en la misma columna indican diferencias estadísticamente significativas $(\alpha=0,05)$

Del cuadro 5 se desprende que el desempeño de los clones es superior al de los testigos de semillas, los cuales alcanzan las menores alturas en los ensayos de Molulco y Remeco y alturas intermedias en el ensayo de la Reserva Forestal Malleco. Como situación excepcional se observa que el testigo T4 presenta la mayor altura promedio en el ensayo de Molulco, pero se trata de unas pocas plantas individuales de gran tamaño inicial al momento de establecer el ensayo, las cuales en su conjunto exhiben una supervivencia tan baja $(8,4 \%)$ que no las hace aconsejables como material de plantación.

El hecho de que los clones de mejor desempeño en cada ensayo sean distintos, sugiere la existencia de interacción genotipo-ambiente. Esta condición puede visualizarse al comparar el ordenamiento o ranking de los clones comunes en los tres ensayos (Figura $N^{\circ} 1$ ), si el ordenamiento fuese similar las línea tendrían tendencia a ser similares también, lo que indicaría que no existe interacción genotipo-ambiente y que los clones con mejor desempeño en un sitio determinado, también estarían entre los mejores en los sitios restantes. No obstante la situación observada es muy heterogénea, por lo mismo, para cada sitio se debe privilegiar un conjunto de clones específicos. 


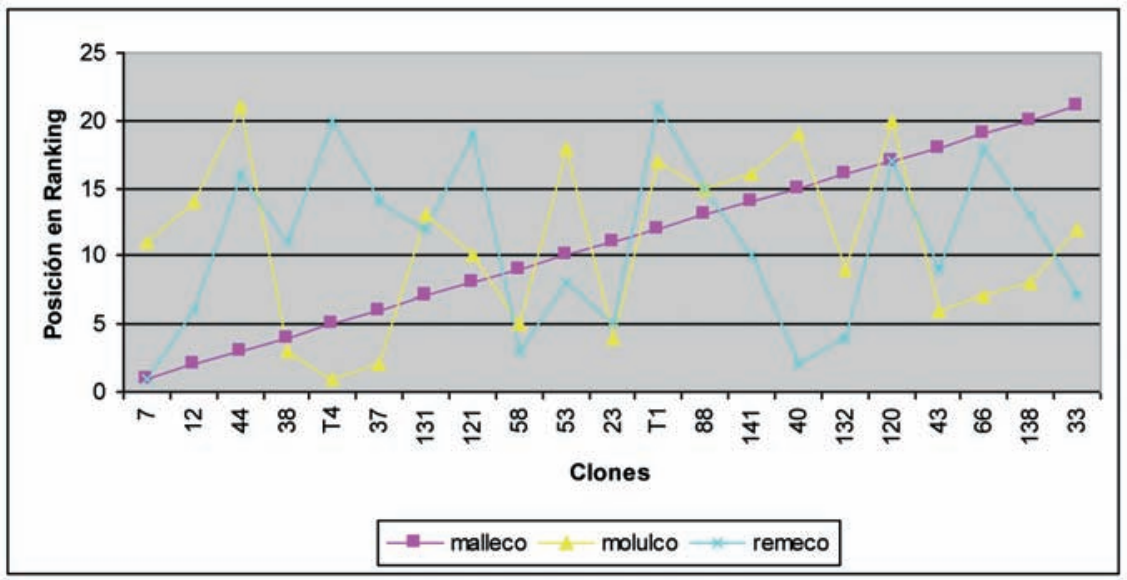

Figura $N^{0} 1$

COMPARACIÓN DE LOS RANKINGS CLONALES EN LOS TRES ENSAYOS EVALUADOS

Tal situación también se corrobora en la matriz de correlación de alturas por clon entre pares de ensayos (Cuadro $\mathrm{N}^{\circ} 6$ ). En la medida que la correlación es positiva y alta no existe interacción genotipo ambiente y los mismos clones son los mejores en todos los sitios. Sin embargo, en la situación observada las correlaciones son bajas, sugiriendo precisamente lo contrario y confirmando la necesidad de usar clones distintos en cada área geográfica representada por los ensayos.

\section{Cuadro $\mathrm{N}^{\circ} 6$}

MATRIZ DE CORRELACIÓN DE ALTURAS ENTRE PARES DE ENSAYOS

\begin{tabular}{|l|c|c|c|}
\hline & RF Malleco & Molulco & Remeco \\
\hline RF Malleco & 1 & 0,11 & 0,08 \\
\hline Molulco & - & 1 & $-0,02$ \\
\hline Remeco & - & - & 1 \\
\hline
\end{tabular}

La existencia de interacción genotipo-ambiente alerta respecto a la inconveniencia de tomar decisiones en base al análisis conjunto o simultáneo de los tres ensayos. Este enfoque, si bien orienta respecto al desempeño relativo medio de los clones evaluados, no permite discriminar cuales son los mejores en cada situación y resulta de interés solo cuando tal interacción no se manifiesta. Por lo mismo el análisis conjunto de los ensayos no se presenta en este documento

En consecuencia, considerando que los sitios ensayados representan tres niveles latitudinales de la distribución natural de raulí en la Cordillera de los Andes, sobre los 800 msnm, el análisis efectuado permite sugerir que en las áreas geográficas comprendidas de norte a sur por las provincias de Malleco, Cautín y Valdivia, los clones más apropiados son los indicados en el Cuadro $\mathrm{N}^{\circ} 7$. 


\section{Cuadro $\mathrm{N}^{\circ} 7$ \\ CLONES Y PROCEDENCIAS DE MEJOR DESEMPEÑO \\ PARA PLANTAR RAULÍ EN DISTINTAS ÁREAS GEOGRÁFICA DE SU DISTRIBUCIÓN NATURAL}

\begin{tabular}{|c|c|}
\hline Área geográfica & Clones \\
\hline Malleco & $7,12,44,38$ \\
\hline Cautín & $98,37,38,23$ \\
\hline Valdivia & $7,40,58,132$ \\
\hline
\end{tabular}

La sugerencia anterior debe ser considerada como preliminar por cuanto el estado de desarrollo de las plantas aún es demasiado incipiente como para hacer recomendaciones definitivas. Lo mismo puede afirmarse respecto a las procedencias de mejor desempeño, resultando una medida prudente privilegiar el uso de material genético seleccionado en procedencias locales para establecer las plantaciones en cada sitio. Efectivamente, las procedencias locales, Jauja en el ensayo de la RF Malleco, y Neltume en el ensayo Remeco, se encuentran entre las de mejor desempeño en sus respectivos ensayos. Sólo en la plantación de Molulco, la procedencia local (Melipeuco) conforma un grupo cuyo comportamiento es inferior al de las mejores procedencias de ese ensayo.

\section{CONCLUSIONES}

Plantas micropropagadas provenientes de árboles plus de raulí evidencian un mejor comportamiento inicial en terreno que otras generadas a partir de semilla comercial, sugiriéndose que esta diferencia obedece al origen genético del material clonal, más que a la forma de producción de las plantas.

Existen evidencias de interacción genotipo ambiente que aconsejan no usar los mismos clones para distintas condiciones de plantación, privilegiándose en cada caso a los que exhiben el mejor desarrollo en cada zona evaluada.

En términos del origen geográfico de los clones evaluados, se sugiere la utilización de procedencias locales mientras no se cuente con información definitiva, obtenida de evaluaciones efectuadas a edades más avanzadas, que permita confirmar el mejor comportamiento de procedencias individuales en cada zona de plantación. 


\section{REFERENCIAS}

Gutiérrez, B.; Bello, A. y Navarrete, M., 2005. Manual de ensayos clonales de raulí. Documento del proyecto FDI 00C7FT-12: "Silvicultura Clonal en Raulí para Aumentar la Productividad de Sitios Forestales en la IX y X Regiones del País”. INFOR. 44p.

Schlatter, J.E.; Gerding, V. y Adriazola, J., 1994. Sistema de ordenamiento de la tierra. Herramienta para la planificación forestal aplicado a las regiones VII, VIII y IX. Serie Técnica, Fac. Cs. For., Univ. Austral de Chile, Valdivia, Chile. 114 p.

Schlatter, J.E.; Gerding, V. y Huber, H., 1995. Sistema de ordenamiento de la tierra. Herramienta para la planificación forestal aplicado a la X Región. Serie Técnica, Fac. Cs. For., Univ. Austral de Chile, Valdivia, Chile. 93 p.

Schlatter, J.E. y Gerding, V., 1995. Método de clasificación de sitios para la producción forestal, ejemplo en Chile. Bosque 16(2): 13 - 20.

Schlatter, J. y Steuer, H., 2005. Selección de sitios para el establecimiento de ensayos clonales de raulí. En: Gutiérrez, B.; Ortiz, O. y Molina, M. (editores). 2005. Clonación de raulí: Estado actual y perspectivas. INFOR-CEFOR-UACH. Concepción, Chile. Pp: 75-103. 